\title{
A sclerostin-based theory for strain-induced bone formation
}

\author{
René F. M. van Oers - Bert van Rietbergen • \\ Keita Ito • Peter A. J. Hilbers • Rik Huiskes
}

Received: 26 May 2010 / Accepted: 18 October 2010 / Published online: 11 November 2010

(C) The Author(s) 2010. This article is published with open access at Springerlink.com

\begin{abstract}
Bone formation responds to mechanical loading, which is believed to be mediated by osteocytes. Previous theories assumed that loading stimulates osteocytes to secrete signals that stimulate bone formation. In computer simulations this 'stimulatory' theory successfully produced loadaligned trabecular structures. In recent years, however, it was discovered that osteocytes inhibit bone formation via the protein sclerostin. To reconcile this with strain-induced bone formation, one must assume that sclerostin secretion decreases with mechanical loading. This leads to a new 'inhibitory' theory in which loading inhibits osteocytes from inhibiting bone formation. Here we used computer simulations to show that a sclerostin-based model is able to produce a load-aligned trabecular architecture. An important difference appeared when we compared the response of the stimulatory and inhibitory models to loss of osteocytes, and found that the inhibitory pathway prevents the loss of trabeculae that is seen with the stimulatory model. Further, we demonstrated with combined stimulatory/inhibitory models that the two pathways can work side-by-side to achieve a load-adapted bone architecture.
\end{abstract}

Keywords Sclerostin - Bone remodeling - Osteocytes · Osteoblasts

\section{Introduction}

It is well known that increased mechanical loading will lead to an increase in bone mass whereas a state of reduced

R. F. M. van Oers · B. van Rietbergen $(\bowtie) \cdot$ K. Ito ·

P. A. J. Hilbers · R. Huiskes

Department of Biomedical Engineering, Eindhoven University of Technology, PO Box 513, 5600 MB Eindhoven, The Netherlands

e-mail: b.v.rietbergen@tue.nl loading will lead to bone resorption, a finding often referred to as 'Wolff's law' (Wolff 1892). These changes in bone mass are the net result of a change in activity of bone forming cells (osteoblasts) and/or bone resorbing cells (osteoclasts). It is generally assumed now that these changes in cell activity are regulated by osteocytes, because they are strategically located within the bone's lacuno-canalicular network (Burger and Klein-Nulend 1999), and because they show a metabolic response to bone loading (Skerry et al. 1989). However, how, exactly, osteocytes regulate osteoblasts and/or osteoclasts is still unknown.

In earlier studies we hypothesized that osteocytes sense strains, and in response send signals to stimulate osteoblasts. Using computer simulation models, we demonstrated that this mechanism can explain the formation of trabecular-like architectures that adapt to changes in loading magnitude and direction (Huiskes et al. 2000; Ruimerman et al. 2005). Although the exact nature of this stimulus was not detailed, we assumed this to be some biochemical messenger molecule, the secretion of which is increased by mechanical loading.

In contrast to this 'stimulatory' model, it was found in recent years that osteocytes inhibit bone formation via the protein sclerostin (Winkler et al. 2003; van Bezooijen et al. 2007). To reconcile this information with strain-induced bone formation, one needs to assume that sclerostin production by osteocytes decreases with mechanical loading. A recent study by Robling et al. (2008) confirms this assumption: in vivo loading of rat and mice forelimbs significantly reduced sclerostin secretion by osteocytes in the loaded forelimb. A new theory for strain-induced bone formation is thus a case of 'minus times minus equals plus': mechanical loading inhibits the inhibition of bone formation.

Although the pathways by which an inhibitory model and a stimulatory model work are very different, their net effect 
could be the same. Both models will result in an increase in bone formation when mechanical loading is high and a decrease in bone formation when loading is low. We therefore expect that the inhibitory model, like the stimulatory model, is able to produce load-aligned trabeculae that adapt to changing loading conditions.

Although the response to mechanical loading might be similar under normal circumstances, differences can be expected in situations where osteocytes are lost or osteocyte signaling is disrupted, for example due to microdamage. Whereas a reduction in osteocytes would lead to decreased bone formation in the stimulatory model, it would lead to increased bone formation in the inhibitory model since in that case less sclerostin is produced to inhibit osteoblast activity. The consequences of this difference in behavior on bone structure and remodeling, however, are hard to predict.

Finally, it should be noted that the stimulatory and inhibitory model are not mutually exclusive. It is possible that osteocytes stimulate osteoblasts at higher loading conditions and inhibit osteoblast activity at lower loading conditions using different biochemical pathways. The consequences of this combined behavior on bone structure and remodeling obviously are even more difficult to predict.

In the present study we aim at clarifying these issues by using a computer simulation approach. The first goal of this study is to investigate if a sclerostin-based inhibitory model will also produce trabecular-like structures that align to the loading conditions. A second goal is to investigate and compare the response of the stimulatory and inhibitory model to the loss of osteocytes. A final goal is to investigate if a combined stimulatory/inhibitory model is viable and whether it would produce similar results.

\section{Methods: the model}

Our sclerostin-based inhibitory model is based on and compared to the stimulatory model by Huiskes et al. (2000). This will be described first, after which we will address the changes made for the inhibitory model and the combined model.

\subsection{The stimulatory model}

A bone structure is mapped onto a finite element mesh consisting of square elements of uniform size $\Delta x$. An element, at location $\boldsymbol{x}$ and at time $t$, has a relative bone density $m(\boldsymbol{x}, t)$ that determines its Young's modulus $E(\boldsymbol{x}, t)$, according to (Currey 1988):

$E(\mathbf{x}, t)=E_{\max } \cdot m(\mathbf{x}, t)^{3}$.

Finite element analysis (FEA) is used to calculate the local mechanical strains resulting from external loads acting on the
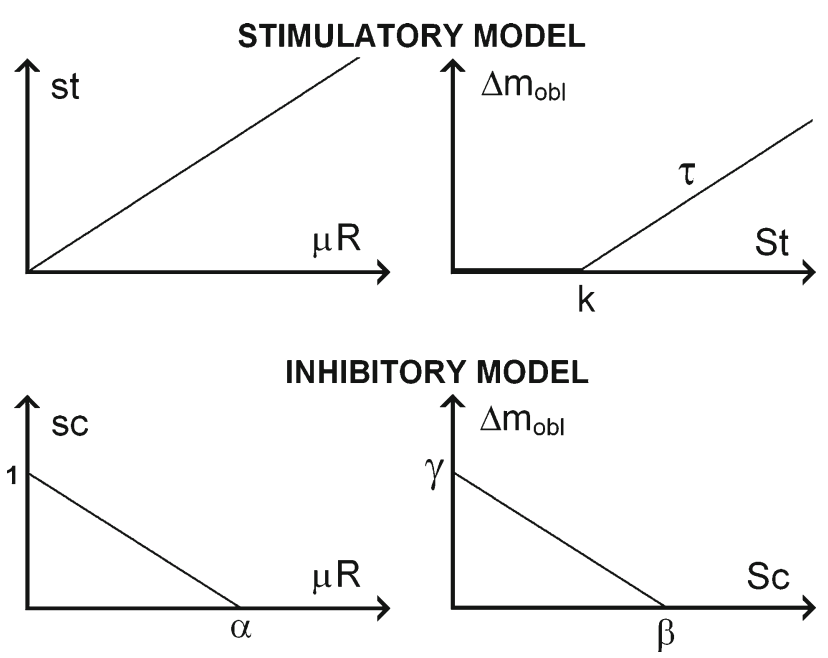

Fig. 1 Relations from osteocyte sensation $(\mu R)$ to bone formation $\left(\Delta m_{\mathrm{obl}}\right)$. In the stimulatory model, mechanical loading $(\sim \mu R)$ stimulates the secretion of a signal $(s t)$ that stimulates bone formation $\left(\Delta m_{\mathrm{obl}}\right)$. In the inhibitory model, mechanical loading $(\sim \mu R)$ inhibits the secretion of a signal (sc) that inhibits bone formation $\left(\Delta m_{\mathrm{obl}}\right)$

bone structure. Osteocytes within the bone (elements with $m(\boldsymbol{x}, t)>0.5)$ are assumed to sense a mechanical stimulus $R$, for which we use the local strain-energy-density (SED) rate as a correlate (Brown et al. 1990; Huiskes 2000). Note that we use a static load to represent a cyclic load of given amplitude $\sigma$ and frequency $f$. The magnitude of the static load $\sigma^{\prime}$ is chosen such that the resulting strain energy density (SED) equals the peak SED rate of the dynamic load:

$\sigma^{\prime}=2.02 \sigma \sqrt{f}$.

For a derivation of this formula, see Huiskes (2000) or Ruimerman et al. (2001).

In the stimulatory model an osteocyte $i$ responds to this mechanosensation by emitting a signal $s t_{i}$ (Fig. 1, top left):

$s t_{i}(t)=R\left(\mathbf{x}_{i}, t\right) \cdot \mu$,

depending on the mechanical stimulus at its location $\boldsymbol{x}_{i}$ and osteocyte mechanosensitivity $\mu$. The signal is assumed to decrease exponentially in strength with increasing distance $d$ from the osteocyte. The exponential function represents the steady-state distribution of a signal molecule, where synthesis and decay are in balance (Lancaster 1996). Each element at the bone surface receives an accumulated signal $S t$ from $n$ nearby osteocytes, according to:

$S t(\mathbf{x}, t)=\sum_{i=1}^{n} s t_{i}(t) \cdot e^{-d\left(\mathbf{x}, \mathbf{x}_{i}\right) / D}$,

where $D$ is a diffusion-decay constant, $\boldsymbol{x}_{i}$ is the position of osteocyte $i$ and $n$ is the number of osteocytes less than $d_{\text {infl }}$ removed from $\boldsymbol{x}$, where $d_{\text {infl }}$ is a truncation distance for the osteocyte signal. Osteoblasts are recruited to bone surfaces 
where the signal exceeds a threshold $k$. They then form bone according to (Fig. 1, top right):

$\Delta m_{\mathrm{obl}}=\tau \cdot(S t(\mathbf{x}, t)-k)$,

where the change in $m(\boldsymbol{x}, t)$ due to osteoblast activity is denoted with the index obl, and $\tau$ determines the bone formation rate.

Osteoclast activity is represented by resorption cavities occurring randomly along the trabecular surface, with a probability $p_{\text {ocl }}$. If an element on the bone surface is subject to osteoclastic resorption, $m(\boldsymbol{x}, t)$ is decreased by an amount $r_{\text {ocl }}$ :

$\Delta m_{\mathrm{ocl}}=-r_{\mathrm{ocl}}$.

An alternative version of Huiskes' model (Huiskes et al. 2000) had osteoclast activity inhibited by strain-induced osteocyte signals, and this was also central to our extended version of the model (van Oers et al. 2008), where osteoclasts were explicitly modeled and guided by osteocyte signals. In this study we have chosen for random resorption, however, to ensure that any adaptation to mechanical loading is the result of osteocyte-osteoblast crosstalk.

\subsection{The inhibitory model}

In the inhibitory model the osteocyte is assumed to secrete a signal (sclerostin) $s c_{i}$ when its mechanical sensation is below a threshold $\alpha$, according to (Fig. 1, bottom left):

$s c_{i}(t)=1-R\left(\mathbf{x}_{i}, t\right) \cdot \mu / \alpha$.

Like the stimulatory signal $s t_{i}$, the inhibitory signal $s c_{i}$ is assumed to decrease in strength with increasing distance. Elements at the bone surface receive an accumulated signal $S c$ :

$S c(\mathbf{x}, t)=\sum_{i=1}^{n} s c_{i}(t) \cdot e^{-d\left(\mathbf{x}, \mathbf{x}_{i}\right) / D}$,

where we assume the same diffusion-decay constant $D$ as for the stimulatory signal. Osteoblasts form bone when $S c$ falls below a threshold $\beta$, according to (Fig. 1, bottom right):

$\Delta m_{\mathrm{obl}}=\gamma \cdot(1-S c(\mathbf{x}, t) / \beta)$,

where $\gamma$ defines the bone formation amount at zero sclerostin.

\subsection{The combined model}

In the combined model we assume that both pathways exist alongside each other, and both the stimulatory signal $s t$ and the inhibitory signal $s c$ are being produced by osteocytes. For the resulting bone formation, we sum the formations of the above two models:

$$
\Delta m_{\mathrm{obl}}=\frac{1}{2} \tau \cdot(S t(\mathbf{x}, t)-k)+\frac{1}{2} \gamma \cdot(1-S c(\mathbf{x}, t) / \beta) .
$$

The two formation components were halved to maintain a formation rate similar to the single pathway models. They can also be combined such that one pathway predominates. We will use one combined model where the stimulatory pathway predominates:

$\Delta m_{\mathrm{obl}}=\frac{3}{4} \tau \cdot(S t(\mathbf{x}, t)-k)+\frac{1}{4} \gamma \cdot(1-S c(\mathbf{x}, t) / \beta)$,

and another where the inhibitory pathway predominates:

$\Delta m_{\mathrm{obl}}=\frac{1}{4} \tau \cdot(S t(\mathbf{x}, t)-k)+\frac{3}{4} \gamma \cdot(1-S c(\mathbf{x}, t) / \beta)$.

We will refer to these three combined models as the (50/50)-, (75/25)-, and (25/75)-combined model, respectively.

2.4 Model adaptation to prevent undesired discretization effects

During preliminary testing of the inhibitory model, we observed an unexpected effect of the discretization. This effect is described and explained in Appendix A. To prevent it we have chosen the following approach: before an element is half-filled $(m(\boldsymbol{x}, t)>0.5)$ its formation rate is determined by the formation rates as calculated for its neighboring bone elements (only counting those with $m\left(\boldsymbol{x}_{n b}, t\right)>0.5$ ).

$\Delta m_{\mathrm{obl}}= \begin{cases}\sum_{n b} \frac{1}{4} \gamma \cdot\left(1-S c\left(\mathbf{x}_{n b}, t\right) / \beta\right) & \text { if } m(\mathbf{x}, t) \leq 0.5 \\ \gamma \cdot(1-S c(\mathbf{x}, t) / \beta) & \text { if } m(\mathbf{x}, t)>0.5\end{cases}$

Although, as Appendix A explains, the effect will not occur in the stimulatory model, for the sake of honest comparison we also introduce the same approach here:

$\Delta m_{\mathrm{obl}}= \begin{cases}\sum_{n b} \frac{1}{4} \tau \cdot\left(S t\left(\mathbf{x}_{n b}, t\right)-k\right) & \text { if } m(\mathbf{x}, t) \leq 0.5 \\ \tau \cdot(S t(\mathbf{x}, t)-k) & \text { if } m(\mathbf{x}, t)>0.5\end{cases}$

and these changes are likewise incorporated in the combined models.

\section{Methods: the simulations}

For all models, trabecular remodeling is simulated in a $4 \times 4 \mathrm{~mm}^{2}$ domain, initially an arbitrary, porous bone lattice (Fig. 2, left). The domain is subjected to a $6.06 \mathrm{MPa}$ compressive load in the vertical direction and a tensile load in the horizontal direction. This is continued for 300 model increments. The loading direction is then changed by $30^{\circ}$, and the structure is allowed to adapt for another 300 increments.

We also investigate the response of these models to a pathological situation with severe osteocyte death. $75 \%$ of osteocytes are removed at random from the previously generated 


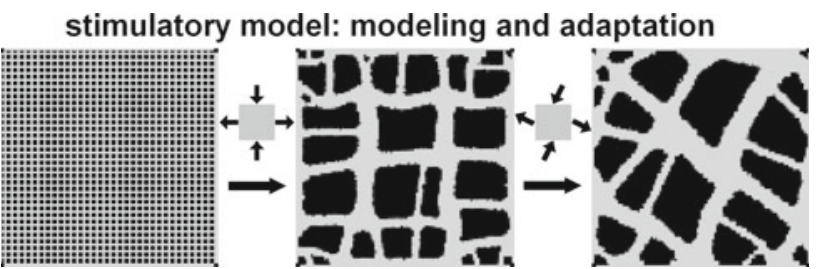

Fig. 2 Trabecular modeling and adaptation in the stimulatory model

Table 1 Parameter settings

\begin{tabular}{llll}
\hline Parameter & Symbol & Value & Unit \\
\hline Element size & $\Delta x$ & 40 & $\mu \mathrm{m}$ \\
Maximum Young's modulus & $E_{\max }$ & 15 & $\mathrm{GPa}$ \\
Poisson's ratio & $\nu$ & 0.3 & - \\
Osteocyte density & $\rho_{\text {ocy }}$ & 1,600 & $\mathrm{~mm}^{-2}$ \\
Osteocyte mechanosensitivity & $\mu$ & 1.0 & $\mathrm{~J}^{-1} \mathrm{~m}^{3}$ \\
Sensation limit for sclerostin secretion & $\alpha$ & $4.0 \times 10^{4}$ & \\
Diffusion-decay constant & $\mathrm{D}$ & 100 & $\mu \mathrm{m}$ \\
Stimulus threshold for bone formation & $k$ & $8.0 \times 10^{4}$ & - \\
Formation to stimulus ratio & $\tau$ & $2.5 \times 10^{-7}$ & - \\
Sclerostin limit for bone formation & $\beta$ & 5.0 & - \\
Formation amount at zero sclerostin & $\gamma$ & 0.3 & - \\
Resorption amount & $r_{\text {ocl }}$ & 0.3 & - \\
Resorption probability & $p_{\text {ocl }}$ & 0.2 & - \\
\hline
\end{tabular}

trabecular structures, and the model is allowed to recover for 200 increments. Newly built bone has a normal osteocyte density. All parameters are given in Table 1.

\section{Results}

As resorption events perforated some of the initial struts, strains in the remaining load-bearing struts increased. In the stimulatory model this induced more secretion of osteocyte signals, which in turn induced bone formation that thickened these struts. Other struts that did not contribute to the main lines of load transfer, were not strengthened by bone formation and got resorbed. Within about 50 increments, the main trabecular architecture was defined. After that a few thin trabeculae were removed, but at 300 increments the structure (Fig. 2, middle) was clearly in equilibrium and well-adapted to its loading conditions. Changing the load direction caused high strains at the trabecular edges closest to the new stress trajectories, which in turn induced rapid bone formation along those edges. Redundant bone at the opposing edges was gradually removed. In this process some trabeculae were lost. The result was a trabecular structure that was aligned to the new loading direction (Fig. 2, right).

In the inhibitory model, low strains in the initial lattice corresponded to sclerostin secretion throughout the structure, which suppressed bone formation. As resorption events inhibitory model: modeling and adaptation

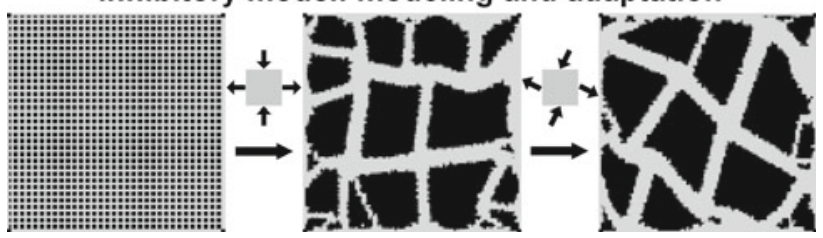

Fig. 3 Trabecular modeling and adaptation in the inhibitory model

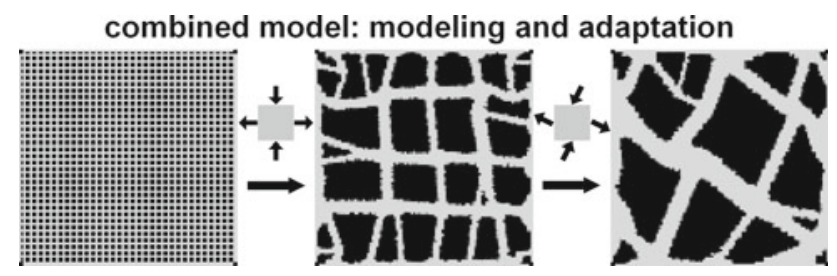

Fig. 4 Trabecular modeling and adaptation in the combined model

disconnected struts, strains increased in the remaining loadbearing struts. This strain increase reduced the local secretion of sclerostin to a level that allowed thickening of these struts. A trabecular architecture (Fig. 3, middle) was formed, aligned to the imposed loading directions. When load directions were changed, the structure adapted accordingly (Fig. 3, right). The transition followed the same pattern as before, starting with rapid bone formation at the trabecular edges closest to the new stress trajectories, followed by the slow removal of redundant bone on opposite edges.

In the (50/50)-combined model both processes described above were at work, resulting in a trabecular architecture (Fig. 4, middle), that adapted when load directions were changed (Fig. 4, right). Similar results were found for the (75/25)- and (25/75)-combined model (results not shown).

When $75 \%$ of osteocytes were removed in the stimulatory model, there was a corresponding reduction in the bone formation stimulus. Resorption thinned all trabeculae. Note that there was no increase in osteoclast activity; thinning occurred because ongoing osteoclast activity now outbalanced the reduced osteoblast activity. As some trabeculae were disconnected, increased strains in the remaining struts induced bone formation. Since the newly formed bone had a normal osteocyte density, these struts rapidly increased in thickness. The resulting structure was one with considerably less, but much thicker trabeculae (Fig. 5, left).

When $75 \%$ of osteocytes were removed in the inhibitory model, there was a corresponding reduction in sclerostin. Bone formation prevailed on all trabecular surfaces. Because this new bone layer was of normal osteocyte density, and because trabecular thickening had reduced the strains, sclerostin secreted from this layer quickly halted further thickening. If resorption events removed part of this layer, the lack of sclerostin often restored it again. The architecture was therefore 


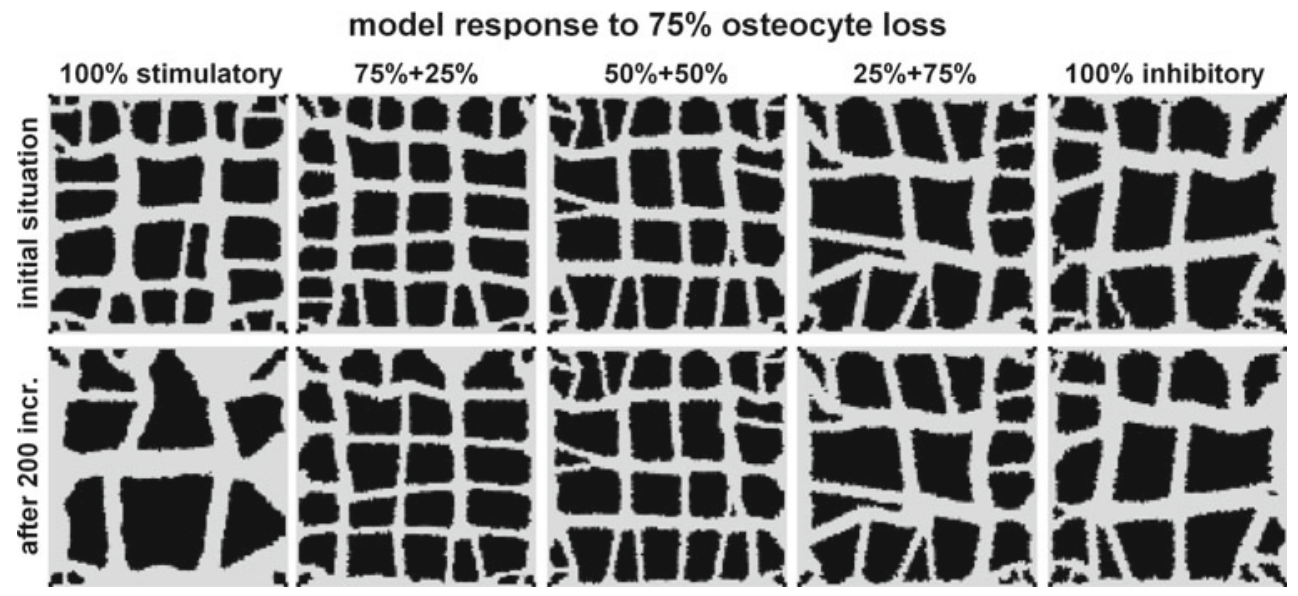

Fig. 5 Response to $75 \%$ osteocyte loss for all five models. In the stimulatory model model resorption prevailed initially and many trabeculae were lost. This effect was seen to a lesser extent in the (75/25)-com-

preserved. After 200 increments, no trabeculae had been lost compared to the initial situation(Fig. 5, right).

In the (75/25)-combined model a few trabeculae were lost (Fig. 5, second column), but much less than in the completely stimulatory model. In the (50/50)-combined model, osteocyte removal had practically no effect. The potential effect that a drop in stimulatory signals could have on bone formation appeared to be compensated by a similar drop in sclerostin. The structure (Fig. 5, middle) was maintained. The (25/75)-model behaved much like the completely inhibitory model and no trabeculae were lost.

\section{Discussion}

Our simulations show that a sclerostin-based model is able to produce a load-aligned trabecular architecture that adapts when the loading conditions are changed. In this context it is necessary that mechanical stimuli reduce sclerostin secretion by osteocytes, as was recently confirmed by Robling et al. (2008) and Papanicolaou et al. (2009). Compared to our previous stimulatory model, where strains stimulate osteocytes to stimulate osteoblasts, this model is the exact reverse: strains inhibit the inhibition of bone formation. The result is just the same.

The real functional implications appear when osteocytes are lost. In the stimulatory model this dramatically reduces bone formation, in the inhibitory model it does the opposite. Consequently, many trabeculae were lost in the stimulatory model, whereas the structure was protected in the inhibitory model. This is not entirely advantageous, since the bone with the dead osteocytes is not renewed. It should be emphasized that we excluded any effects of osteocyte death on osteoclast activity. In this study we chose for random resorption, to ensure that any adaptation to mechanical load resulted from bined model. In the other combined models and the inhibitory model the structure was preserved

osteocyte-osteoblast crosstalk, be it via the stimulatory or the inhibitory pathway. However, it is believed that osteoclasts target dead osteocytes for resorption (Bentolila et al. 1998; Verborgt et al. 2000). If the stimulatory model were combined with such targeted resorption, loss of osteocytes would result in an even greater loss of trabeculae than was seen here. If the inhibitory model were combined with targeted resorption, loss of osteocytes could induce temporary bone formation (a microcallus) on the outside of the trabecula combined with resorption of the dead osteocytes within. This combination has a better potential to preserve trabecular connectivity during removal of the dead bone.

Likewise, the two models would show different behavior when osteocytes are absent from the start, such as in the remodeling of scaffolds or the turnover of calcified cartilage. The stimulatory model predicts little bone formation in such cases, whereas the inhibitory model predicts the exact opposite. Recently, Cox et al. (2011) used computer simulations based on the stimulatory model to study the replacement of calcified cartilage by bone under the growth plate. They found that osteocytes at the bone-cartilage interface were able to facilitate this turnover via the stimulatory pathway. Nevertheless, the process might be facilitated even more via the inhibitory pathway, which would promote bone formation further from the bone-cartilage interface.

The simulations with the combined models also produced a trabecular-like architecture that aligned to the loading conditions. It must be mentioned however, that the two pathways were combined simply by adding their effects. Without precise knowledge of both pathways, and where they act on the osteoblast cellular events leading to bone formation, this approach seems reasonable. What the combined model shows is that the regulation mechanism does not need to be either stimulatory or inhibitory, but can include both pathways. Regarding the response to osteocyte loss, the addition 
of the inhibitory pathway prevented loss of trabeculae, even in the $(75 / 25)$-combined model where the stimulatory pathway predominated.

The resorption pits in this study have no explicit shape, as in the original study by Huiskes et al. (2000). Later studies have explicitly modeled osteoclasts resorbing lacunae of several elements (van Oers et al. 2008), or lacunae with an explicit shape and size of several elements (Liu et al. 2008). Although it would be possible to use the latter models here, we believe it would not offer any advantages for the present research goals.

For the new inhibitory model, we assume that the secretion of sclerostin is inhibited by mechanical loading. A simple linear decrease in relation to strain-energy-density (SED) is used to capture this assumption, whereas in actual bone this might be a nonlinear decrease in relation to strain-induced fluid flow. On the receiving side, the osteoblastic reaction to osteocytic sclerostin is also implemented with a simple linear relation. Note that the older stimulatory pathway was also implemented with simple linear relations. We did not intend to present the precise physiological parameter values for these pathways here. We intended to demonstrate that, when mechanical loading "stimulates the stimulation" and "inhibits the inhibition" of bone formation, load-aligned structures can be produced. Whatever the precise relation, if mechanical loading were to increase sclerostin secretion, this would not facilitate the emergence of a load-aligned bone architecture. We further intended to demonstrate that these two pathways react differently to osteocyte loss. Whether osteoblast reaction to the stimulatory and inhibitory signals is linear or non-linear, this difference remains.

Currently the inhibitory pathway via sclerostin receives a lot of attention, but there are also several studies pointing to stimulatory pathways (Heino et al. 2004; Imai et al. 2009; Tatsumi et al. 2007; Taylor et al. 2007; Vezeridis et al. 2006). Tatsumi et al. (2007) were able to specifically kill 70-80\% of osteocytes in mice, via a toxin that did not directly affect other bone cells; although they observed a marked decrease in sclerostin mRNA, they did not observe an increase in osteoblast activity, which lead them to suggest that "it is conceivable that in addition to negative regulators such as sclerostin, one or more positive regulators are produced by osteocytes". Taylor et al. (2007) co-cultured osteocytes exposed to fluid flow with osteoblasts shielded from this flow but connected to the osteocytes via gap junctions: fluid flow on osteocytes led to increased alkaline phosphatase activity in osteoblasts, but not if gap junctions were blocked. This indicates that there was a stimulatory signal reaching the osteoblasts via gap junctions. These stimulatory signals have not been identified yet. Prostaglandins have been implicated (Chow and Chambers 1994; Burger and Klein-Nulend 1999), but their role is unclear (Suponitzky and Weinreb 1998; Taylor et al. 2007), and may have to do more with regulating osteocyte gap junctions (Cheng et al. 2001), than directly stimulating osteoblast activity.

Further, there may be more inhibitory pathways than only via sclerostin. Robling et al. (2008) showed that the expression of dickkopf homolog 1 (Dkk1), another inhibitor of bone formation, also decreased with mechanical loading. Although our inhibitory model was inspired by sclerostin, the same concept could thus apply to other inhibitors of bone formation. The existence of multiple stimulatory and inhibitory pathways only makes the system more robust. As the combined model has shown, these pathways can work side-by-side to achieve a load-adapted bone architecture.

Acknowledgments This work was supported by the Netherlands Organization for Scientific Research, section Computational Life Sciences (NWO/CLS, grant number 635.100.014).

Open Access This article is distributed under the terms of the Creative Commons Attribution Noncommercial License which permits any noncommercial use, distribution, and reproduction in any medium, provided the original author(s) and source are credited.

\section{Appendix A: discretization effect in the inhibitory model}

During preliminary testing of the inhibitory model, an unexpected effect was observed. The emerging trabeculae were covered with multiple protrusions, as shown in Fig. 6.

We believe that this is not inherent to the proposed regulation mechanism, but a model artifact, which is explained in Fig. 7. Imagine for a moment that there would be such protrusions on a real trabecula (Fig. 7, left). These protrusions would be unloaded, in contrast to the center of the trabecula. Hence, more sclerostin should be produced within these protrusions, halting formation at their surfaces (indicated by - ). Surfaces near the center of the trabecula (indicated by + ) on the other hand, should receive less

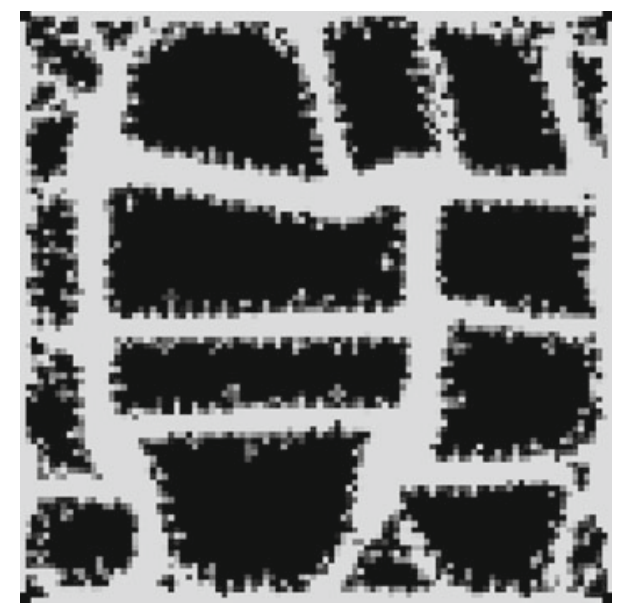

Fig. 6 Trabeculae covered with protrusions in the inhibitory model 

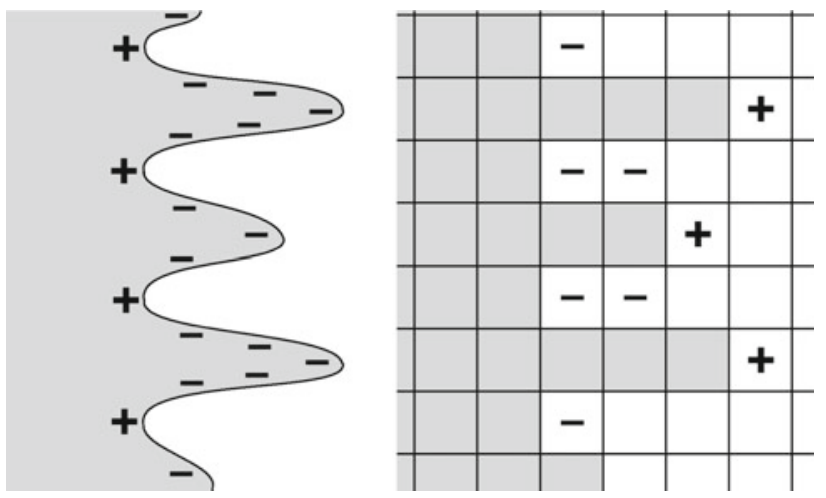

Fig. 7 Discretization of the sclerostin based model allows more formation $(+)$ at the tips of protrusions than at their bases

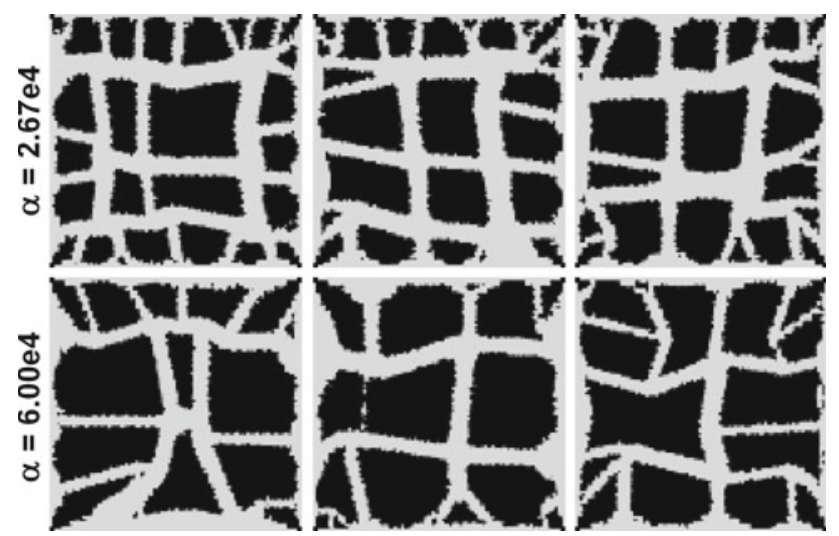

Fig. 8 Simulation results with low $\alpha$ (above) and high $\alpha$ (below)

sclerostin, allowing formation there. As a result the protrusions would be expected to disappear. Now if we look at a discretized trabecula (Fig. 7, right), bone formation is not determined per bone surface, but per element near the bone surface. All elements around the protrusions receive sclerostin. In between two protrusions, the sclerostin level is strongest, inhibiting bone formation (indicated by -). At the protrusion tips, sclerostin levels are slightly weaker, allowing formation (indicated by + ). Due to this discretization effect, the protrusions are maintained. Our approach to prevent it, given in the methods section, could be summarized as follows: the surface element still gets formation as long as one of its neighbor bone elements has low sclerostin (Fig. 8).

Note that such protrusions would never be formed or maintained in the stimulatory model: here osteocyte signals are produced in the load-bearing center of the trabecula, stimulating formation at the base of such protrusions, rather than at the tips.

The protrusions in the inhibitory model are also partly explained by the way the model represents the diffusion of the osteocyte signal. We use an exponential signal-decrease function that represents the steady-state distribution of a

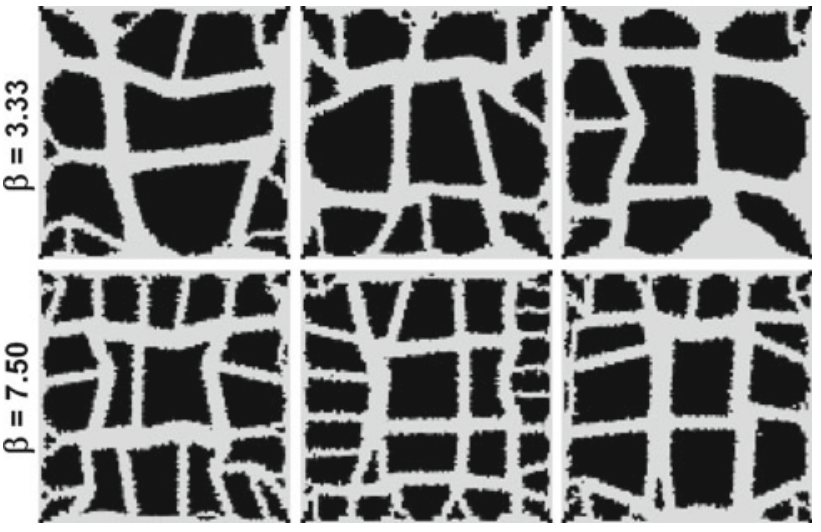

Fig. 9 Simulation results with low $\beta$ (above) and high $\beta$ (below)

signal molecule, where synthesis and decay are in balance (Lancaster 1996). The signal-decrease function makes no difference whether the signal diffuses through bone or marrow. It is conceivable that the transition from canaliculi to marrow space has a significant effect on the diffusion profile. Can sclerostin from an unloaded 'protrusion', after leaving the canaliculi, just diffuse through marrow space and inhibit bone formation on another nearby bone surface? The protrusions effect may also be remedied with a better model for signal diffusion, that takes the effective diffusivity through canaliculi and marrow space into account.

\section{Appendix B: influence of parameters $\alpha$ and $\beta$}

With the inhibitory model we introduced parameters $\alpha$ and $\beta$, where $\alpha$ determines a level of mechanosensation that would halt sclerostin secretion by osteocytes, and $\beta$ determines a sclerostin concentration that would halt bone formation by osteoblasts. Note that these parameters were not yet based on experimental data, although they may be in the future. The model is still conceptual, and uses simple linear equations between mechanical loading, sclerostin secretion, and bone formation, which could be given with these few parameters.

We have, however, investigated how variations in the values of these parameters influence the outcome of the inhibitory model. The figure below shows three simulation results with a lower $\alpha$ (factor 2/3), and three with a higher $\alpha$ (factor $3 / 2$ ). When $\alpha$ was increased, the mechanosensation needed to halt sclerostin production was higher, so bone formation remained inhibited at higher mechanical loading. Consequently, the resulting bone structure lost more trabeculae with higher $\alpha$.

When the sclerostin limit for bone formation $\beta$ was increased, more sclerostin was needed to halt bone formation. Consequently, the resulting bone structure retained more trabeculae for higher $\beta$ (Fig. 9, below). 


\section{References}

Bentolila V, Boyce TM, Fyhrie DP, Drumb R, Skerry TM, Schaffler MB (1998) Intracortical remodeling in adult rat long bones after fatigue loading. Bone 23:275-281

Brown TD, Pedersen DR, Gray ML, Brand RA, Rubin CT (1990) Toward an identification of mechanical parameters initiating periosteal remodeling: a combined experimental and analytic approach. J Biomech 23:893-905

Burger EH, Klein-Nulend J (1999) Mechanotransduction in bone-role of the lacuno-canalicular network. FASEB J 13 Suppl:101-112

Cheng B, Kato Y, Zhao S, Luo J, Sprague E, Bonewald LF, Jiang JX (2001) $\mathrm{PGE}_{2}$ is essential for gap junction-mediated intercellular communication between osteocyte-like MLO-Y4 cells in response to mechanical strain. Endocrinology 142:3464-3473

Chow JW, Chambers TJ (1994) Indomethacin has distinct early and late actions on bone formation induced by mechanical stimulation. Am J Physiol Endocrinol Metab 267:E287-E292

Cox LGE, van Rietbergen B, van Donkelaar CC, Ito K (2011) The turnover of mineralized growth plate cartilage into bone may be regulated by osteocytes. J Biomech (accepted)

Currey JD (1988) The effect of porosity and mineral content on the Young's modulus of elasticity of compact bone. J Biomech 21:131-191

Heino TJ, Hentunen TA, Väänänen HK (2004) Conditioned medium from osteocytes stimulates the proliferation of bone marrow mesenchymal stem cells and their differentiation into osteoblasts. Exp Cell Res 294:458-468

Huiskes R (2000) If bone is the answer, then what is the question? J Anat 197:145-156

Huiskes R, Ruimerman R, van Lenthe GH, Janssen JD (2000) Effects of mechanical forces on maintenance and adaptation of form in trabecular bone. Nature 405:704-706

Imai S, Heino TJ, Hienola A, Kurata K, Büki K, Matsusue Y, Väänänen HK, Rauvala H (2009) Osteocyte-derived HB-GAM (pleiotrophin) is associated with bone formation and mechanical loading. Bone 44:785-794

Lancaster J (1996) Diffusion of free nitric oxide. Methods Enzymol 268:31-50

Liu XS, Huang AH, Zhang XH, Sajda P, Baohua J, Guo XE (2008) Dynamic simulation of three dimensional architecture and mechanical alterations in human trabecular bone during menopause. Bone 43:292-301

Papanicolaou SE, Phipps RJ, Fyhrie DP, Genetos DC (2009) Modulation of sclerostin expression by mechanical loading and bone morphogenetic proteins in osteogenic cells. Biorheology 46:389-399

Robling AG, Niziolek PJ, Baldridge LA, Condon KW, Allen MR, Alam I, Mantila SM, Gluhak-Heinrich J, Bellido TM, Harris SE, Turner
CH (2008) Mechanical stimulation of bone in vivo reduces osteocyte expression of Sost/sclerostin. J Biol Chem 283:5866-5875

Ruimerman R, Hilbers PAJ, van Rietbergen B, Huiskes R (2005) A theoretical framework for strain-related trabecular bone maintenance and adaptation. J Biomech 38:931-941

Ruimerman R, Huiskes R, van Lenthe GH, Janssen JD (2001) A computer-simulation model relating bone-cell metabolism to mechanical adaptation of trabecular architecture. Comput Methods Biomech Biomed Eng 4:433-448

Skerry TM, Bitensky L, Chayen J, Lanyon LE (1989) Early strainrelated changes in enzyme activity in osteocytes following bone loading in vivo. J Bone Miner Res 4:783-788

Suponitzky I, Weinreb M. (1998) Differential effects of systemic prostaglandin $E_{2}$ on bone mass in rat long bones and calvariae. J Endocrinol 156:51-57

Tatsumi S, Ishii K, Amizuka N, Li M, Kobayashi T, Kohno K, Ito M, Takeshita S, Ikeda K (2007) Targeted ablation of osteocytes induces osteoporosis with defective mechanotransduction. Cell Metab 5:464-475

Taylor AF, Saunders MM, Shingle DL, Cimbala JM, Zhou Z, Donahue HJ (2007) Mechanically stimulated osteocytes regulate osteoblastic activity via gap junctions. Am J Physiol Cell Physiol 292:545552

van Bezooijen RL, Svensson JP, Eefting D, Visser A, van der Horst G, Karperien M, Quax PH, Vrieling H, Papapoulos SE, ten Dijke P, Löwik CW (2007) Wnt but not BMP signaling is involved in the inhibitory action of sclerostin on BMP-stimulated bone formation. J Bone Miner Res 22:19-28

van Oers RFM, Ruimerman R, Tanck E, Hilbers PAJ, Huiskes $\mathrm{R}$ (2008) A unified theory for osteonal and hemi-osteonal remodeling. Bone 42:250-259

Verborgt O, Gibson GJ, Schaffler MB (2000) Loss of osteocyte integrity in association with microdamage and bone remodeling after fatigue in vivo. J Bone Miner Res 15:60-67

Vezeridis PS, Semeins CM, Chen Q, Klein-Nulend J (2006) Osteocytes subjected to pulsating fluid flow regulate osteoblast proliferation and differentiation. Biochem Biophys Res Commun 348:10821088

Winkler DG, Sutherland MK, Geoghegan JC, Yu C, Hayes T, Skonier JE, Shpektor D, Jonas M, Kovacevich BR, Staehling-Hampton K, Appleby M, Brunkow ME, Latham JA (2003) Osteocyte control of bone formation via sclerostin, a novel BMP antagonist. EMBO J 22:6267-6276

Wolff J (1892) Das Gesetz der Transformation der Knochen. Hirschwald, Berlin; translated as: Wolff J (1986) The law of bone remodeling. Springer, Berlin 